\title{
Near Field and Far Field Analysis of Alternating Impedance Electromagnetic Bandgap (AI-EBG) Structure for Mixed-Signal Applications
}

\author{
Jinwoo Choi ${ }^{1}$, Dong Gun $\mathrm{Kam}^{2}$, Daehyun Chung ${ }^{1,2}$, Krishna Srinivasan ${ }^{1}$, Vinu Govind ${ }^{1}$, Joungho Kim ${ }^{2}$, and \\ Madhavan Swaminathan ${ }^{1}$ \\ ${ }^{1}$ School of Electrical and Computer Engineering, Georgia Institute of Technology \\ Atlanta, Georgia 30332, USA \\ E-mail: jwchoi@ece.gatech.edu and madhavan.swaminathan@ece.gatech.edu \\ ${ }^{2}$ Terahertz Interconnection and Package Laboratory, Department of Electrical Engineering, \\ Korea Advanced Institute of Science and Technology (KAIST), Daejeon 305-701, Korea
}

\begin{abstract}
This paper presents near field $(N F)$ and far field $(F F)$ analysis of alternating impedance electromagnetic bandgap (AI$E B G)$ structure in package and board. Three test vehicles have been designed and fabricated for near field and far field measurements. Simulation results using a full wave solver (SONNET ${ }^{T M}$ ) have been compared with measurement results. This paper investigates the radiation due to return current on different reference planes. The analysis results from simulations and measurements provide important guidelines for design of the AI-EBG structure for noise reduction in mixed-signal systems.
\end{abstract}

\section{Introduction}

Electromagnetic bandgap (EBG) structures have been suggested for noise reduction in high-speed digital and mixed-signal systems in recent years due to their characteristics of suppression of unwanted electromagnetic mode transmission [1]-[5]. Among these EBG structures, alternating impedance electromagnetic bandgap (AI-EBG) structure has shown good noise suppression in mixed-signal systems [2]-[5]. Fig. 1 shows the measured LNA output spectrum around $2.1 \mathrm{GHz}$ for the mixedsignal systems with and without the AI-EBG structure [4]. The $7^{\text {th }}$ harmonic noise peak at $2.1 \mathrm{GHz}$ was suppressed completely from $-58 \mathrm{dBm}$ to $-88 \mathrm{dBm}$ using the AI-EBG structure. Fig. 2 shows the dispersion diagram of the AI-EBG structure in the mixed-signal system, which shows passbands and stopbands. Since $2.1 \mathrm{GHz}$ is a frequency in the stopband, as shown in Fig. 2, noise generated from digital circuits can not propagate to a RF circuit (an LNA in this case) at this frequency.

However, none of the previous works [2]-[5] has discussed the radiated emission from the AI-EBG structures. The AI-EBG structure has periodic gaps that can radiate energy if the AI-EBG structure is used as a reference plane. This is because return currents are forced to flow around the splits and charges therefore accumulate on their edges [6], which creates a source of far field radiation [7]. Hence, the splits or gaps in a reference plane can be considered as slot antennas that can interfere with other devices, which causes an electromagnetic interference (EMI) problem. In this paper, the surface radiation from the AI-EBG structures due to return current has been quantified.

In this paper, near field and far field analysis for three test vehicles were carried out through simulations and measurements to better understand the radiation mechanisms of the AI-EBG structure.

\section{Design of three test vehicles}

Three test vehicles have been designed and fabricated for radiation analysis. First test vehicle is a microstrip line on a solid plane, the second test vehicle is a microstrip line on an AI-EBG structure, and the third test vehicle is a microstrip line on an embedded AI-EBG structure. The third test vehicle was designed to suppress noise in mixed-signal systems without any EMI problems. This is possible since the solid plane was used as a reference plane for the microstrip line in this embedded AI-EBG structure. In Fig. 3, the cross section of these three test vehicles are shown. The top view of these three test vehicles is also shown in Fig. 4. The dielectric material of the test vehicles is FR4 with a relative permittivity, $\varepsilon_{\mathrm{r}}=4.4$, the conductor is copper with conductivity, $\sigma_{\mathrm{c}}=5.8 \times 10^{7} \mathrm{~S} / \mathrm{m}$, and dielectric loss tangent is $\tan (\delta)=0.02$. The copper thickness for microstrip line, solid plane and AI-EBG plane in the test vehicles is $35 \mu \mathrm{m}$, dielectric thickness between two conductors is 5 mils and the dielectric thickness of the most bottom layer is 28 mils. For the AI-EBG structures in the second and third test vehicles, the size of the metal patch is $1.5 \mathrm{~cm} \times 1.5 \mathrm{~cm}$ and the size of metal branch is $0.1 \mathrm{~cm} \times 0.1 \mathrm{~cm}$, which is shown in Fig. 7. It should be noted that the size of the metal patches in the first column near SMA connector is $1.3 \mathrm{~cm} \times 1.5 \mathrm{~cm}$.

\section{Near field (NF) simulation and measurement}

The return current on the reference plane of a microstrip line plays an important role in near-field electromagnetic coupling. Hence, the return current effects should be considered in package and board design [7]. The full wave solver (SONNET ${ }^{\mathrm{TM}}$ ) was used for near field simulation of the three test vehicles. To make sure that simulation results are correct, the standing wave patterns on the reference plane for the test vehicle 1 were captured and these patterns are shown in Fig. 5. Standing waves are formed at $800 \mathrm{MHz}, 1.6 \mathrm{GHz}$, and $2.4 \mathrm{GHz}$ for the test vehicle 1 . Two frequencies at $300 \mathrm{MHz}$ and $2.7 \mathrm{GHz}$ were chosen for the near field analysis. Fig. 6 shows current density for the solid reference plane in the test vehicle 1 at $300 \mathrm{MHz}$ and $2.7 \mathrm{GHz}$ and Fig. 7 shows current density for the AI-EBG plane in the test vehicle 2. The current densities for the solid reference plane in test vehicle 3 (an embedded AI-EBG structure), which are not shown in this paper, showed the same results as those for the 
test vehicle 1 since a solid plane was used for both test vehicles. It should be noted that current densities at $300 \mathrm{MHz}$ for the test vehicles 1 and 2 showed the same tendency but, at $2.7 \mathrm{GHz}$, the current density for the AI-EBG plane in the test vehicle 2 showed a non-ideal current flow, which can cause higher radiation.

The near field measurement was carried out using EMC Precision Scan (EPS-3000) equipment and NEC probe (CP-25). Generally, near field measurement in the $\mathrm{GHz}$ range is very difficult since almost all of the commercially available probes are not sensitive for near field measurement in $\mathrm{GHz}$ range. However, the NEC probe in this paper covers a bandwidth of $3 \mathrm{GHz}$. Fig. 8 shows near field measurement results for the test vehicle 1 at $300 \mathrm{MHz}$ and $2.7 \mathrm{GHz}$ while Fig. 9 shows near field measurement results for the test vehicle 2 at $300 \mathrm{MHz}$ and $2.7 \mathrm{GHz}$. In these near field measurements, y component of magnetic field $\left(\mathrm{H}_{\mathrm{y}}\right)$ was recorded and the unit of the magnetic field intensity is $[\mathrm{dB} \mu \mathrm{V}]$. The near field measurement results for the test vehicle 3 are shown in Fig. 10. Test vehicle 1 and 3 showed the same results since a solid plane was used as a reference plane for both cases. For test vehicle 2, near field results at $300 \mathrm{MHz}$ showed the same results as those for test vehicles 1 and 3 at $300 \mathrm{MHz}$ but near field results at $2.7 \mathrm{GHz}$ showed totally different results from those for test vehicles 1 and 3 since an AIEBG plane was used as a reference plane for the test vehicle 2, which causes a non-ideal return current flow due to periodic gaps in the AI-EBG plane, which makes magnetic field distribution in an AI-EBG plane different from that in a solid plane. It should be noted that there are similarities between near field simulation results and measurement results.

\section{Far field (FF) simulation and measurement}

The far field simulation was also performed using SONNET ${ }^{\mathrm{TM}}$ for the three test vehicles. In this simulation, surface radiation from the surface of the test vehicles was investigated by changing the degrees ( $\mathrm{phi}=0^{\circ} \sim 180^{\circ}$ at every $10^{\circ}$ and theta $=-$ $90^{\circ} \sim 90^{\circ}$ at every $10^{\circ}$ ). Fig. 11 shows far field simulation results for the three test vehicles. It should be noted that test vehicle 2 showed the maximum radiation intensity (after $2 \mathrm{GHz}$ ) among three test vehicles since an AI-EBG plane was used as a reference plane for the test vehicle 2 and a periodic pattern in the AI-EBG plane makes higher radiation in a stopband.

To verify the above simulation results, far field measurements were done for the test vehicles. The far field measurements were carried out using Anritsu MG3642A RF signal generator (BW: $125 \mathrm{kHz} \sim 2,080 \mathrm{MHz}$ ), Agilent E4440A spectrum analyzer (BW: $3 \mathrm{kHz} \sim 26.5 \mathrm{GHz}$, Res. $\mathrm{BW}=$ Video $\mathrm{BW}=3 \mathrm{MHz}$ ), and antenna in anechoic chamber. Fig. 12 (a) shows the measurement set-up for the far field measurements. Since the RF signal generator works properly up to $2 \mathrm{GHz}$, the far field measurement was also done up to $2 \mathrm{GHz}$. The distance between EUT and antenna was $3 \mathrm{~m}$ in this case. The RF signal generator was connected to EUT as a source and the spectrum analyzer, which was connected to the antenna, recorded the field intensity from the surface of the test vehicles. In this measurement, radiation intensity from test vehicle 2 is the maximum among three test vehicles, as shown in Fig. 12 (b), and test vehicles 1 and 3 showed almost the same radiation intensity because a solid plane was used as a reference plane for these two test vehicles. It should be noted that the radiated power intensities of the far field measurements in Fig. 12 (b) are in the range of the simulated power intensities in Fig. 11, except for the peaks at $190 \mathrm{MHz}$ and $550 \mathrm{MHz}$ for test vehicle 2.

\section{Conclusion}

In this paper, near field and far field analysis of the AI-EBG structure for mixed signal applications have been demonstrated. Three test vehicles were analyzed for radiation through near field and far field simulations and measurements. Test vehicle 2 (an AI-EBG plane as a reference plane) showed the maximum radiation intensity in near and far field simulations and measurements among three test vehicles, which could cause a possible EMI problem. To minimize a possible EMI problem, the test vehicle with an embedded AI-EBG structure (test vehicle 3) was designed and showed almost the same (or a little better) radiation characteristics as that of test vehicle 1 (reference test vehicle). This test vehicle (test vehicle 3 ) showed that an embedded AI-EBG structure can be used to suppress noise in mixed-signal system without a possible EMI problem.

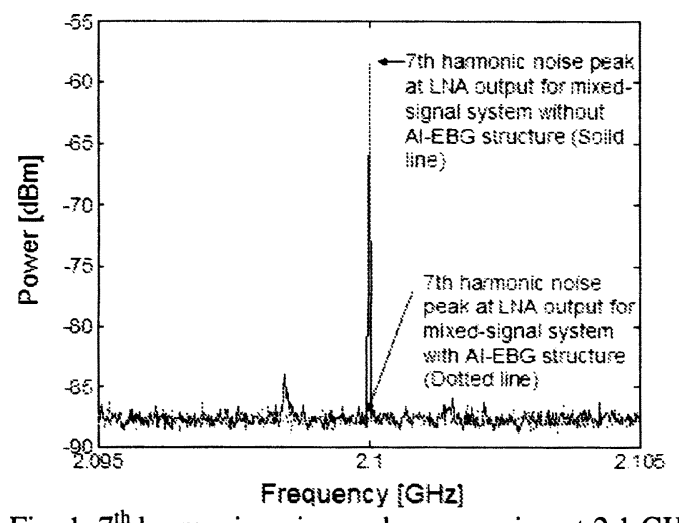

Fig. $1.7^{\text {th }}$ harmonic noise peak suppression at $2.1 \mathrm{GHz}$ in the mixed-signal system with the AI-EBG structure.

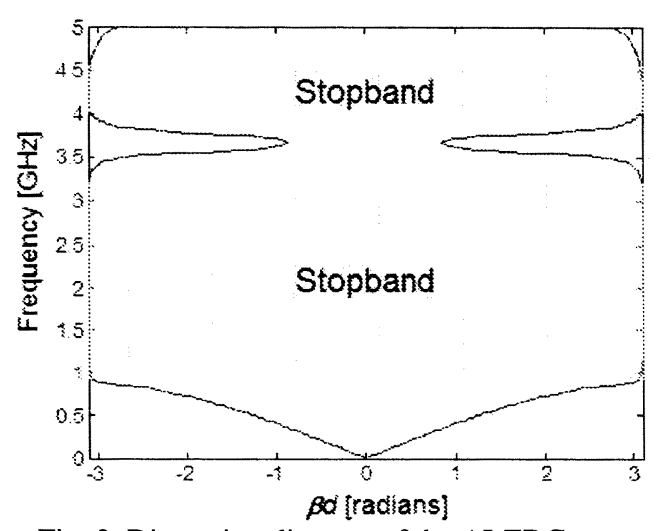

Fig. 2. Dispersion diagram of the AI-EBG structure in the mixed-signal system. 


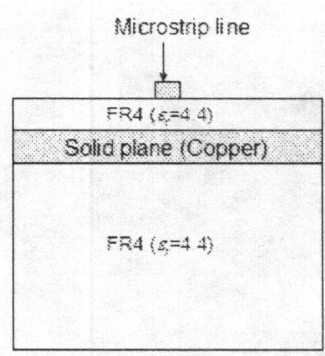

(a)

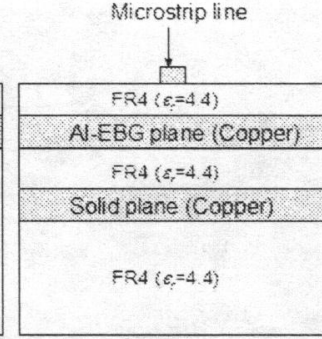

(b)

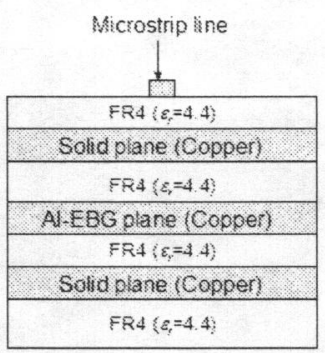

(c)

Fig. 3. Cross section of the three test vehicle. (a) Test vehicle 1 is a microstrip line on a solid plane. (b) Test vehicle 2 is a microstrip line on an AI-EBG structure. (c) Test vehicle 3 is a microstrip line on an embedded AI-EBG structure.

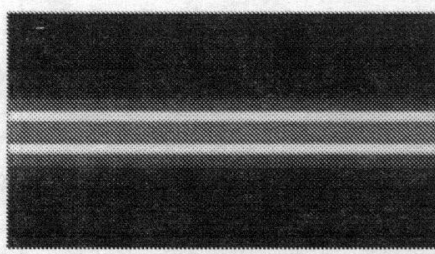

(a)

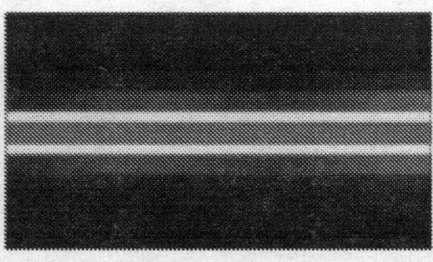

(b)

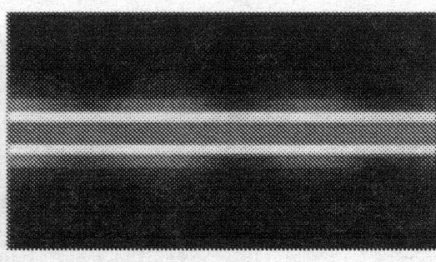

(c)

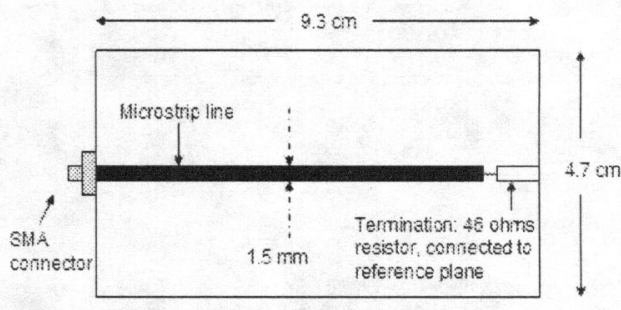

Fig. 4. Top view of the test vehicles.

Fig. 5. Standing wave patterns on reference plane of the test vehicle 1. (a) $1^{\text {st }}$ resonance pattern at $800 \mathrm{MHz}$. (b) $2^{\text {nd }}$ resonance pattern at $1.6 \mathrm{GHz}$. (c) $3^{\text {rd }}$ resonance pattern at $2.4 \mathrm{GHz}$.

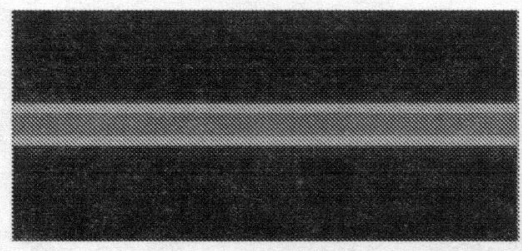

(a)

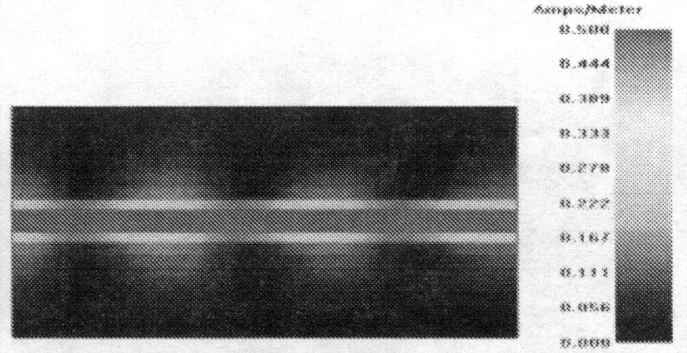

(b)

Fig. 6. Near field simulation results for the test vehicle 1. (a) Current density at $300 \mathrm{MHz}$. (b) Current density at $2.7 \mathrm{GHz}$.
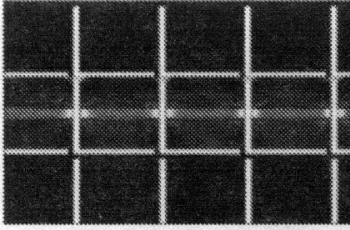

(a)

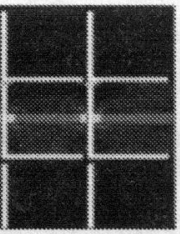

.
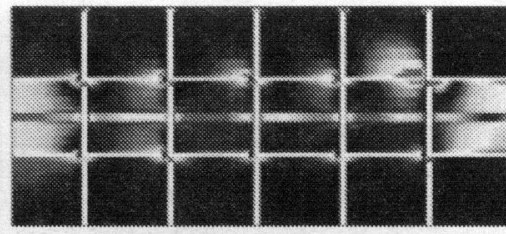

(b)

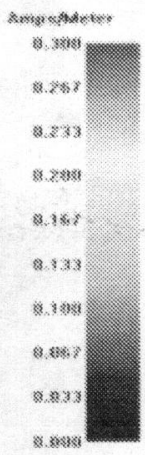

Fig. 7. Near field simulation results for the test vehicle 2. (a) Current density at $300 \mathrm{MHz}$. (b) Current density at $2.7 \mathrm{GHz}$.

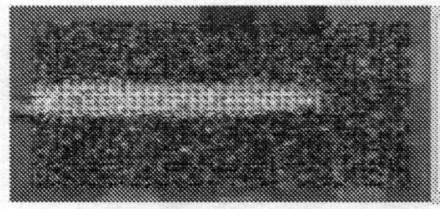

(a)

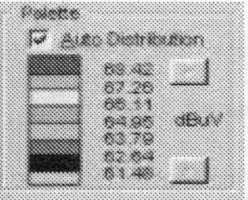

Fig. 8. Near field measurement results for the test vehicle 1. (a) Magnetic field intensity at $300 \mathrm{MHz}$. (b) Magnetic field intensity at $2.7 \mathrm{GHz}$. 


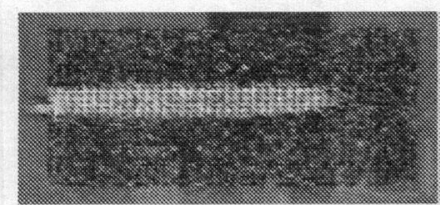

(a)

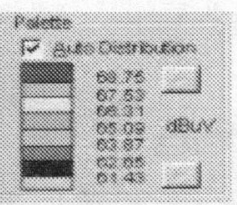

Fig. 9. Near field measurement results for the test vehicle 2. (a) Magnetic field intensity at $300 \mathrm{MHz}$. (b) Magnetic field intensity at $2.7 \mathrm{GHz}$.

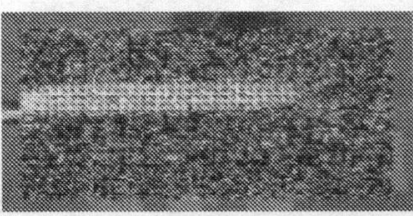

(a)

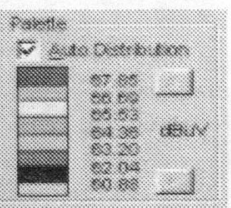

(1)

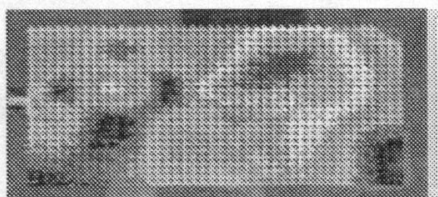

(b)

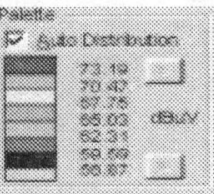

2.

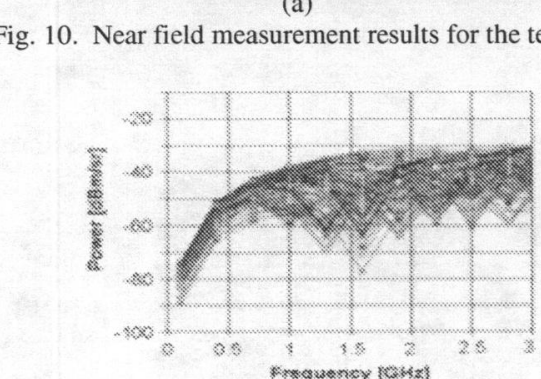

(a)

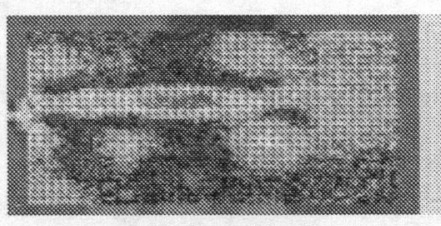

(b)

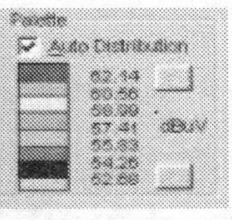

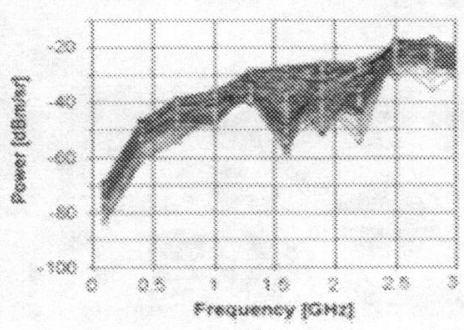

(b)

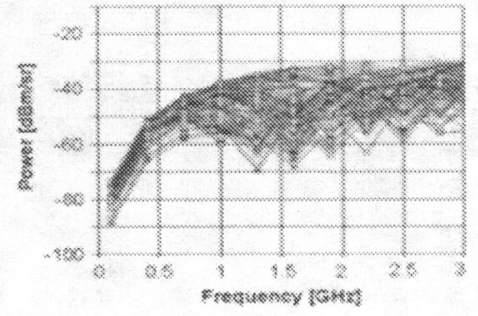

(c)

Fig. 11. Far field simulation results. (a) Test vehicle 1 (a solid plane as a reference plane). (b) Test vehicle 2 (an AI-EBG plane as a reference plane). (c) Test vehicle 3 (a solid plane in an embedded AI-EBG structure as a reference plane).

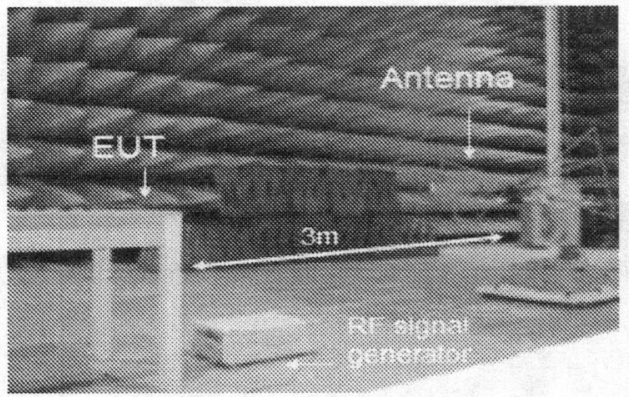

(a)

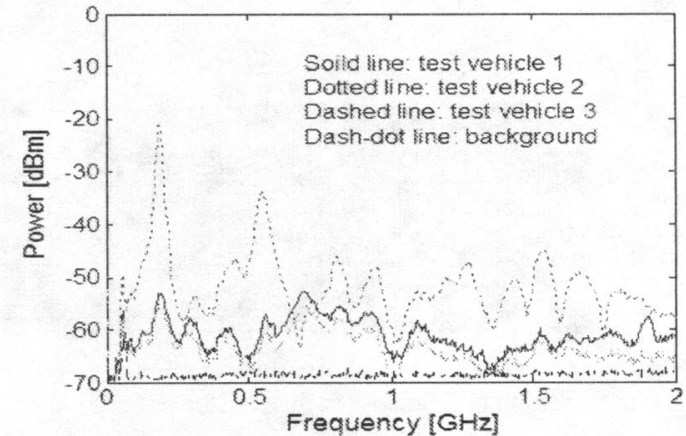

(b)

Fig. 12. Far field measurement set-up and results. (a) Measurement set-up for far field. (b) Far field measurement results.

\section{References}

[1] T. Kamgaing and O. Ramahi, "High-impedance electromagnetic surfaces for parallel-plate mode suppression in high-speed digital systems" IEEE $11^{\text {th }}$ Topical Meeting on Electrical and Performance of Electronic Packaging (EPEP), pp. 279-282, Monterey, CA, Oct. 2002.

[2] J. Choi, V. Govind, and M. Swaminathan, "A novel electromagnetic bandgap (EBG) structure for mixed-signal system applications," IEEE Radio and Wireless Conference (RAWCON), Atlanta, GA, pp.243-246, Sep. 2004.

[3] J. Choi, V. Govind, M. Swaminathan, L. Wan, and R. Doraiswami, "Isolation in mixed-signal systems using a novel electromagnetic bandgap (EBG) structure," IEEE 13 $3^{\text {th }}$ Topical Meeting on EPEP, Portland, Oregon, pp. 199-202, Oct. 2004.

[4] J. Choi, V. Govind, R. Mandrekar, S. Janagama, and M. Swaminathan, "Noise reduction and design methodology for the mixed-signal systems with alternating impedance electromagnetic bandgap (AI-EBG) structure," IEEE MTT-S International Microwave Symposium (IMS), Long Beach, California, June 2005.

[5] J. Choi, V. Govind, and M. Swaminathan, "Noise suppression in ultra wide band (UWB) applications using alternating impedance electromagnetic bandgap (AI-EBG) structure," accepted for presentation at $35^{\text {th }}$ European Microwave Conference (EuMC), Paris, France, Oct. 2005.

[6] T. E. Moran, K. L. Virga, G. Aguirre, and J. L. Prince, "Methods to reduce radiation from split ground planes in RF and mixed signal packaging structures," IEEE Trans. Advanced Packaging, vol.25, no.3, pp. 409-416, Aug. 2002.

[7] P. Fornberg, A. Byers, M. Piket-May, and C. Holloway, "FDTD modeling of printed circuit board signal integrity and radiation," IEEE International Symposium on Electromagnetic Compatibility, pp. 307-312, Aug. 2000.

[8] K. Srinivasan, H. Sasaki, M. Swaminathan, and R. Tummala, "Calibration of near field measurements using microstrip line for noise predictions," Proceedings of Electronic Components and Technology, Las Vegas, Nevada, June 2004. 